Gennadiy Averkov $\cdot$ Johannes Hofscheier · Benjamin Nill

\title{
Generalized flatness constants, spanning lattice polytopes, and the Gromov width
}

\author{
Received: 2 July 2021 / Accepted: 18 November 2021 /
}

Published online: 31 December 2021

\begin{abstract}
In this paper we motivate some new directions of research regarding the lattice width of convex bodies. We show that convex bodies of sufficiently large width contain a unimodular copy of a standard simplex. Following an argument of Eisenbrand and Shmonin, we prove that every lattice polytope contains a minimal generating set of the affine lattice spanned by its lattice points such that the number of generators (and the lattice width of their convex hull) is bounded by a constant which only depends on the dimension. We also discuss relations to recent results on spanning lattice polytopes and how our results could be viewed as the beginning of the study of generalized flatness constants. Regarding symplectic geometry, we point out how the lattice width of a Delzant polytope is related to upper and lower bounds on the Gromov width of its associated symplectic toric manifold. Throughout, we include several open questions.
\end{abstract}

Key words. Lattice polytopes - Spanning lattice polytopes - Lattice width · Flatness constant $\cdot$ Gromov width $\cdot$ Symplectic toric manifolds

\section{Overview}

In this paper we discuss open problems related to the lattice width (and generalizations) motivated by questions on lattice polytopes and symplectic manifolds. Let us shortly explain how this paper is organized and where to find its main results. In Sect. 2 we present applications and questions emerging from Theorem 2.1, that gives an upper bound on the width of lattice polytopes that that do not contain unimodular copies of the standard simplex. In this context, we discuss 'small' lattice-generating subsets (Theorem 2.6) and show that lattice polytopes of arbitrarily large width are not necessarily very ample (Theorem 2.10 ). Open questions

G. Averkov: BTU Cottbus-Senftenberg, Platz der Deutschen Einheit 1, 03046 Cottbus, Germany

e-mail: averkov@b-tu.de

J. Hofscheier: School of Mathematical Sciences, University of Nottingham, Nottingham NG7 2RD, UK

e-mail: johannes.hofscheier@nottingham.ac.uk

B. Nill $(\varangle)$ : Faculty of Mathematics, Otto-von-Guericke-Universität Magdeburg, Universitätsplatz 2, 39106 Magdeburg, Germany

e-mail: benjamin.nill@ovgu.de

Mathematics Subject Classification: Primary: 52B20 · Secondary: 53D05 
are collected in Sect. 2.6. In Sect. 3 we investigate the relations of the Gromov width of symplectic toric manifolds to the lattice width of their moment polytopes. As a consequence of Theorem 2.1 we can give a lower bound on the Gromov width in terms of the lattice width (Corollary 3.2), while on the other hand we formulate the conjecture that the lattice width is always an upper bound for the Gromov width (Conjecture 3.12). Finally, Sects. 4 and 5 contain the proofs of Theorems 2.1 and 2.10 .

\section{The main results from the viewpoint of the geometry of numbers}

\subsection{Convex bodies of large lattice width}

In order to formulate the main result of this section, let us fix some notation. Recall that a non-empty compact and convex subset $K \subseteq \mathbb{R}^{d}$ is called a convex body. The width of a convex body $K \subseteq \mathbb{R}^{d}$ with respect to a non-zero linear functional $\mathbf{u} \in\left(\mathbb{R}^{d}\right)^{*}=\operatorname{Hom}\left(\mathbb{R}^{d}, \mathbb{R}\right)$ is given as

$$
\operatorname{width}_{\mathbf{u}}(K):=\max _{x, y \in K}|\mathbf{u}(x)-\mathbf{u}(y)|,
$$

and the (lattice) width ${ }^{1}$ of $K$ is defined as

$$
\operatorname{width}(K):=\min _{\mathbf{u} \in\left(\mathbb{Z}^{d}\right)^{*} \backslash\{\mathbf{0}\}} \operatorname{width}_{\mathbf{u}}(K),
$$

where $\left(\mathbb{Z}^{d}\right)^{*}=\operatorname{Hom}\left(\mathbb{Z}^{d}, \mathbb{Z}\right)$ denotes the dual lattice.

Here is the classical definition of the flatness constant in dimension $d$ :

$$
\operatorname{Flt}(d):=\sup \left\{\operatorname{width}(K): K \subseteq \mathbb{R}^{d} \text { convex body, } K \cap \mathbb{Z}^{d}=\emptyset\right\} .
$$

It is known that Flt $(d) \leq O\left(d^{\frac{4}{3}} \log ^{a} d\right)$ for a constant $a$ by Rudelson [37] (see also [11]). An explicit upper bound of order $O\left(d^{\frac{5}{2}}\right)$ is given by Flt $(d) \leq \sqrt{\frac{(d+1)(2 d+1)}{6}} d^{\frac{3}{2}}$ [7, Theorem (7.4), (8.3)]. Clearly, Flt(1) $=1$ while in higher dimensions it is known that $\operatorname{Flt}(2)=1+\frac{2}{\sqrt{3}}$ by [28] and $\operatorname{Flt}(3) \leq 3.972$ by [3]. The most recent result known to the authors is [14, Conjecture 1.2] where it is conjectured that Flt $(3)=2+\sqrt{2}$. In [3] evidence is provided in support for this conjecture by finding a local maximizer (with respect to the Hausdorff distance), which attains the conjectured bound such that all other polytopes in a small neighbourhood have strictly smaller width.

Let us recall some more notation. The set $\operatorname{GL}(d, \mathbb{Z})$ consists of the $d \times d$ matrices with integer coefficients and determinant \pm 1 . We will call a map $T: \mathbb{R}^{d} \rightarrow$ $\mathbb{R}^{d}, \mathbf{x} \mapsto A \mathbf{x}+\mathbf{b}$ with $A \in \mathrm{GL}(d, \mathbb{Z})$ and $\mathbf{b} \in \mathbb{Z}^{d}$ an (affine) unimodular transformation, respectively, for $\mathbf{b} \in \mathbb{R}^{d}$ an (affine) $\mathbb{R}$-unimodular transformation. For

1 The reader should be aware that there are other notions sometimes called 'lattice width' in the literature such as the facet width (used in Sect. 3.3) or the max-facet width $[6,36]$. 
$X \subseteq \mathbb{R}^{d}$, we call $T(X)$ a unimodular copy, respectively, an $\mathbb{R}$-unimodular copy of $X$.

We define the $d$-dimensional standard simplex as

$$
\Delta_{d}:=\operatorname{conv}\left(\mathbf{0}, \mathbf{e}_{1}, \ldots, \mathbf{e}_{d}\right)
$$

where $\mathbf{e}_{1}, \ldots, \mathbf{e}_{d}$ is the standard basis of $\mathbb{R}^{d}$. Here is our main observation.

Theorem 2.1. Let $K \subseteq \mathbb{R}^{d}$ be a convex body.

(1) If width $(K) \geq 2 \mathrm{Flt}(d) d$, then $K$ contains a unimodular copy of the standard simplex.

(2) If width $(K) \geq \operatorname{Flt}(d) d$, then $K$ contains an $\mathbb{R}$-unimodular copy of the standard simplex.

The proof will be given in Sect. 4. Let us note the following version of Theorem 2.1(2) (apply it to $\frac{\operatorname{Flt}(d) d}{\operatorname{width}(K)} \cdot K$ ).

Corollary 2.2. Any convex body $K \subseteq \mathbb{R}^{d}$ contains an $\mathbb{R}$-unimodular copy of $\frac{\text { width }(K)}{\operatorname{Flt}(d) d} \cdot \Delta_{d}$.

Remark 2.3. Theorem 2.1 is wrong when one only allows translations of the standard simplex instead of unimodular copies. For instance, consider in dimension two any large multiple of the standard triangle. By shearing it horizontally sufficiently far (by the unimodular transformation $\mathbf{e}_{1} \mapsto \mathbf{e}_{1}, \mathbf{e}_{2} \mapsto k \mathbf{e}_{1}+\mathbf{e}_{2}$ for some large positive integer $k$ ), we get a triangle of the same large lattice width such that every vertical line intersects it in a segment of length $<1$. Hence, this triangle, which is itself a unimodular copy of a multiple of the standard triangle, does not contain a translation of the standard triangle.

\subsection{Lattice-generating subsets of bounded size}

To a lattice polytope $P$, one associates the semigroup of lattice points in $\mathbb{Z}^{d+1}$ in the cone over $P \times\{1\}$. In this case, there is a unique minimal set of generators of this semigroup, called its Hilbert basis (we refer to the book [9] as a pointer to the extensive literature on this topic). Clearly, the size of the Hilbert basis does not accept a bound which only depends upon the dimension. However, if one replaces this semigroup by the subgroup of $\mathbb{Z}^{d+1}$ generated by the lattice points of $P \times\{1\}$, then Theorem 2.6 below shows that the size of a minimal generating subset of the lattice points of $P \times\{1\}$ is bounded by a function in the dimension.

In $[10,40]$, the Carathéodory rank of a cone is defined as the minimal $n \in \mathbb{N}$ such that every lattice point in the semigroup of lattice points in the cone can be written as a nonnegative integral combination of at most $n$ Hilbert basis elements. In [22], Gubeladze extends the Carathéodory rank to polytopes by considering the cone over the polytope and then reducing the study to the cone case. Passing from nonnegative integers to all integral numbers, we give the following related definitions (where we use the linear instead of the affine setting in order to stress the analogy). 
Definition 2.4. Let $P \subseteq \mathbb{R}^{d}$ be a $d$-dimensional lattice polytope, and $\Lambda \subseteq \mathbb{Z}^{d+1}$ the lattice spanned by the lattice points in $P \times\{1\}$.

- The integer linear rank $\operatorname{ILR}(P)$ of $P$ is defined as the minimal $n \in \mathbb{N}$ such that every lattice point in $\Lambda$ can be written as an integer combination of at most $n$ lattice points in $P \times\{1\}$.

- The integer linear complexity $\operatorname{ILC}(P)$ of $P$ is defined as the minimal size of a lattice generating set of $\Lambda$ contained in $P \times\{1\}$.

Similar to the flatness constant Flt $(d)$, we introduce the (maximal) integer linear complexity rank in dimension $d, \operatorname{ILC}(d)$, as the maximal integer linear complexity $\operatorname{ILC}(P)$ of $d$-dimensional lattice polytopes (it follows from Theorem 2.6 below that this definition is well-defined). Analogously, we define $\operatorname{ILR}(d)$.

Remark 2.5. In the previous definition, we adopt notation and terminology that were independently introduced in $[1,2]$. There $\operatorname{ILR}(A)=\operatorname{ILR}(P)$ and $\operatorname{ILC}(A)=$ $\operatorname{ILC}(P)$, where $A$ is the matrix whose columns are the lattice points of $P \times\{1\}$.

We remark that $\operatorname{ILR}(P) \leq \operatorname{ILC}(P) \leq(d+1) \operatorname{ILR}(P)$ (for the second inequality consider a lattice basis of $\Lambda$ ). Clearly, $\operatorname{ILC}(1)=2$ and ILC $(2)=3$ where the second equality follows from the fact that empty lattice triangles are unimodular copies of the standard triangle (recall that a lattice polytope is called empty if the vertices are its only lattice points). In dimension $d=3$, it can be seen from [15, Theorem 1.7] that $\operatorname{ILC}(3)=5$ (and $\operatorname{ILR}(3)=5$ ). By taking lattice pyramids, it follows that $\operatorname{ILC}(d) \geq \operatorname{ILR}(d) \geq d+2$ for $d \geq 3$.

We point out that an exponential bound on $\operatorname{ILR}(d)$ (and thus on $\operatorname{ILC}(d)$ ) can be deduced from an argument of Eisenbrand and Shmonin [18]:

Theorem 2.6. $\operatorname{ILR}(d) \leq 2^{d+1}$.

Proof. For an arbitrary $d$-dimensional lattice polytope $P \subseteq \mathbb{R}^{d}$ we show $\operatorname{ILR}(P) \leq$ $2^{d+1}$. Let $p_{1}, \ldots, p_{m}$ be all lattice points of $P \times\{1\}$ and $\Lambda$ be the lattice generated by $p_{1}, \ldots, p_{m}$. Consider an arbitrary $p \in \Lambda$. We choose a representation $p=$ $\sum_{i=1}^{m} \lambda_{i} p_{i}$ of $p$ (with $\lambda_{1}, \ldots, \lambda_{m} \in \mathbb{Z}$ ), for which the sum

$$
S:=\sum_{i=1}^{m}\left\|\lambda_{i} p_{i}\right\|
$$

of the Euclidean norms of the vectors $\lambda_{1} p_{1}, \ldots, \lambda_{m} p_{m}$ is minimized. We claim that at most $2^{d+1}$ of the coefficients $\lambda_{1}, \ldots, \lambda_{m}$ are not equal to zero. Assume the contrary. Then there are more than $2^{d}$ positive coefficients or more than $2^{d}$ negative coefficients. Both cases are similar, so assume that there are more than $2^{d}$ positive coefficients. This implies the existence of coefficients $\lambda_{s}, \lambda_{t}>0$ with $s \neq t$ for which the respective $p_{s}$ and $p_{t}$ are congruent modulo 2 . This means $\left(p_{s}+p_{t}\right) / 2 \in \mathbb{Z}^{d+1}$ so that $\left(p_{s}+p_{t}\right) / 2=p_{k}$ for some $k \in\{1, \ldots, m\}$. Making both $\lambda_{s}$ and $\lambda_{t}$ smaller by 1 and $\lambda_{k}$ larger by 2 , the sum $S$ gets smaller. Indeed, the triangle inequality implies 


$$
\begin{aligned}
& \left\|\left(\lambda_{s}-1\right) p_{s}\right\|+\left\|\left(\lambda_{t}-1\right) p_{t}\right\|+\left\|\left(\lambda_{k}+2\right) p_{k}\right\| \leq\left\|\left(\lambda_{s}-1\right) p_{s}\right\| \\
& \quad+\left\|\left(\lambda_{t}-1\right) p_{t}\right\|+\left\|\lambda_{k} p_{k}\right\|+\left\|2 p_{k}\right\| \\
& \quad<\left\|\left(\lambda_{s}-1\right) p_{s}\right\|+\left\|\left(\lambda_{t}-1\right) p_{t}\right\|+\left\|\lambda_{k} p_{k}\right\|+\left\|p_{s}\right\|+\left\|p_{t}\right\| \\
& \quad=\left\|\lambda_{s} p_{s}\right\|+\left\|\lambda_{t} p_{t}\right\|+\left\|\lambda_{k} p_{k}\right\| .
\end{aligned}
$$

This yields a contradiction to the choice of $\lambda_{1}, \ldots, \lambda_{m}$ and proves the assertion.

It is not clear if the bound $\operatorname{ILR}(d) \leq 2^{d+1}$ is tight asymptotically. The bound $\operatorname{ILR}(d) \leq 2^{d+1}$ implies $\operatorname{ILC}(d) \leq(d+1) 2^{d+1}$. By Theorem 2.1, the convex hull of any lattice generating set of $\Lambda$ of $\operatorname{size} \operatorname{ILC}(P)$ has width bounded by $2 \operatorname{Flt}(d) d$. Moreover, also the width of $d$-dimensional lattice polytopes $P$ (for $d \geq 3$ ) satisfying $\operatorname{ILC}(P)=\operatorname{ILC}(d)$ is bounded by $2 \operatorname{Flt}(d) d$.

\subsection{Bounding the lattice width of non-spanning lattice polytopes}

A lattice polytope $P \subseteq \mathbb{R}^{d}$ is called spanning if every lattice point in $\mathbb{Z}^{d}$ is an affine integral combination of lattice points in $P$. In the notation of Definition 2.4, this is equivalent to $\Lambda=\mathbb{Z}^{d+1}$. Spanning lattice polytopes are in the focus of current research in classifications and Ehrhart theory of lattice polytopes as they form a large class of lattice polytopes that have nice Ehrhart-theoretic properties [15,24, 25]. On the other hand, non-spanning lattice polytopes are quite exceptional, for instance, they include the much-studied class of empty lattice simplices (excluding unimodular copies of the standard simplex). In [15] building upon [13], a complete classification of all non-spanning three-dimensional lattice polytopes was achieved. In particular, it follows from their main result ([15, Theorem 1.3]) that the width of a non-spanning lattice polytope of dimension $d=3$ is at most 3 (compare this with the maximal width 1 of empty lattice tetrahedra). Here, it follows from Theorem 2.1(1) that such an upper bound exists in any dimension. ${ }^{2}$

Corollary 2.7. Any $d$-dimensional lattice polytope $P \subseteq \mathbb{R}^{d}$ with width $(P) \geq$ $2 \mathrm{Flt}(d) d$ is spanning.

Remark 2.8. Containing a unimodular copy of the standard simplex is in general stronger than being spanning, however, at least in lower dimensions surprisingly not by much. Theorem 1.7 in [15] shows that there are only two spanning lattice 3 -polytopes that do not contain a unimodular copy of the standard simplex.

We remark that the more classical situation of large dilations (instead of large width) is much easier and completely understood.

Proposition 2.9. Let $P \subseteq \mathbb{R}^{d}$ be a d-dimensional lattice polytope. Then $k P$ is spanning for $k \geq\left\lfloor\frac{d+1}{2}\right\rfloor$.

${ }^{2}$ It has been recently proven by Ambro and Ito [4, Theorem 5.2(c)] using algebrogeometric methods that one can replace $\operatorname{Flt}(d)$ in Corollary 2.7 by $d$. 
Proof. We may assume that $P$ is a lattice simplex (otherwise triangulate $P$ and use one of the simplices in the triangulation). Consider the closed parallelepiped $\Pi$ spanned by $P \times\{1\} \subseteq \mathbb{R}^{d+1}$ (see e.g. [12]). The lattice $\mathbb{Z}^{d+1}$ is spanned by all the lattice points in $\Pi$ which includes the vertices $\mathbf{v}_{0}, \ldots, \mathbf{v}_{d}$ of $P \times\{1\}$. As $\Pi$ has the symmetry $\mathbf{x} \mapsto \mathbf{v}_{0}+\cdots+\mathbf{v}_{d}-\mathbf{x}$, we see that $\mathbb{Z}^{d+1}$ is already spanned by all the lattice points in $\Pi$ with last coordinate $\leq \frac{d+1}{2}$. From this the statement follows.

The previous bound is sharp in any odd dimension $d \geq 3$ : consider for instance the unique empty lattice simplex of normalized volume 2 (e.g., see [27]).

\subsection{Further properties of lattice polytopes of large lattice width?}

It is tempting to conjecture that lattice polytopes of large width satisfy even stronger properties than spanning such as IDP (sometimes also called integrally-closed) or very ample (we refer to [9] for the precise definitions). However, this is not true.

Theorem 2.10. For any dimension $d \geq 3$ and any integer $k \geq 3$ there exists a $d$-dimensional lattice polytope $P \subseteq \mathbb{R}^{d}$ of width $k$ such that for any integer $t \geq 2$ there is a lattice point in $t P$ that is not the sum of $t$ lattice points in $P$.

The proof will be given in Sect. 5 .

\subsection{Generalized flatness constants}

Finally, we would like to rephrase Theorem 2.1 in terms of generalized flatness constants, as this seems to us a promising unifying approach to several of the above questions.

Definition 2.11. For a bounded subset $X \subseteq \mathbb{R}^{d}$, we define the flatness constant with respect to $X$ by

$$
\begin{aligned}
& \mathrm{Flt}_{d}(X):=\sup \\
& \text { \}width }(K): K \subseteq \mathbb{R}^{d} \text { convex body, } K \text { does not contain a unimodular copy of } X\{,
\end{aligned}
$$

and the $\mathbb{R}$-flatness constant with respect to $X$ by

$$
\begin{aligned}
& \mathrm{Flt}_{d}^{\mathbb{R}}(X):=\sup \\
& \quad \text { width }(K): K \subseteq \mathbb{R}^{d} \text { convex body, } \\
& \quad K \text { does not contain an } \mathbb{R} \text {-unimodular copy of } X\{.
\end{aligned}
$$

For $X=\{\mathbf{0}\} \subseteq \mathbb{R}^{d}$, we recover the usual flatness constant, i.e.,

$$
\operatorname{Flt}_{d}(\{\mathbf{0}\})=\operatorname{Flt}(d) .
$$

We remark that $\mathrm{Flt}_{d}^{\mathbb{R}}(X) \leq \mathrm{Flt}_{d}(X)$, and both generalized flatness constants are monotone with respect to inclusion. Flt $_{d}$ is invariant under unimodular transformations while $\mathrm{Flt}_{d}^{\mathbb{R}}$ is invariant under $\mathbb{R}$-unimodular transformations. Moreover, it is 
straightforward to show that $\mathrm{Flt}_{d}^{\mathbb{R}}(n X)=n \mathrm{Flt}_{d}^{\mathbb{R}}(X)$ for any positive real number $n$ while the analogous statement for $\mathrm{Flt}_{d}(\cdot)$ is a priori not clear.

Theorem 2.1 implies that these generalized flatness constants are real numbers.

Corollary 2.12. Let $X \subseteq \mathbb{R}^{d}$ be a bounded subset which fits in a unimodular copy of $n \cdot \Delta_{d}$. Then $\mathrm{Flt}_{d}(X) \leq 2 n d \cdot \operatorname{Flt}(d)$ and $\mathrm{Flt}_{d}^{\mathbb{R}}(X) \leq n d \cdot \operatorname{Flt}(d)$.

Let us show how determining $\mathrm{Flt}_{d}^{\mathbb{R}}(X)$ would allow to find the order of $\mathrm{Flt}_{d}(n$. $X$ ), cf. Questions 6 and 7 below. The following lemma will be crucial in this explanation:

Lemma 2.13. For any $X \subseteq \mathbb{R}^{d}$, we have $\operatorname{Flt}_{d}(X) \leq \operatorname{Flt}_{d}^{\mathbb{R}}\left(X+[0,1]^{d}\right)$.

Proof. We show that any convex body $K \subseteq \mathbb{R}^{d}$ which contains an $\mathbb{R}$-unimodular copy of $X+[0,1]^{d}$ does also contain a unimodular copy of $X$. Suppose $A \cdot(X+$ $\left.[0,1]^{d}+\mathbf{b}\right) \subseteq K$ for some $A \in \mathrm{GL}_{d}(\mathbb{Z})$ and $\mathbf{b} \in \mathbb{R}^{d}$. We can write $\mathbf{b}=\mathbf{b}^{\prime}-\mathbf{b}^{\prime \prime}$ for $\mathbf{b}^{\prime} \in \mathbb{Z}^{d}$ and $\mathbf{b}^{\prime \prime} \in[0,1]^{d}$. Then $A \cdot\left(X+\mathbf{b}^{\prime}\right) \subseteq A \cdot\left(X+\mathbf{b}+[0,1]^{d}\right) \subseteq K$, i.e., $K$ contains a unimodular copy of $X$.

Remark 2.14. In general the inequality in the previous lemma is strict. For example, $\operatorname{Flt}_{1}\left(\left\{\frac{1}{3}\right\}\right)=\frac{2}{3}$ while $\operatorname{Flt}_{1}^{\mathbb{R}}\left(\left\{\frac{1}{3}\right\}+[0,1]\right)=1$.

Suppose that $X \subseteq \mathbb{R}^{d}$ is a full-dimensional convex body, so that there is $\lambda>0$ such that an $\mathbb{R}$-translate of $\lambda^{-1}[0,1]^{d}$ is contained in $X$. Then by the previous lemma, we obtain

$$
\begin{aligned}
& n \cdot \mathrm{Flt}_{d}^{\mathbb{R}}(X)=\mathrm{Flt}_{d}^{\mathbb{R}}(n \cdot X) \leq \mathrm{Flt}_{d}(n \cdot X) \leq \mathrm{Flt}_{d}^{\mathbb{R}}\left(n \cdot X+[0,1]^{d}\right) \\
& \quad \leq \mathrm{Flt}_{d}^{\mathbb{R}}((n+\lambda) \cdot X)=(n+\lambda) \cdot \mathrm{Flt}_{d}^{\mathbb{R}}(X) .
\end{aligned}
$$

We divide this inequality by $n$ and get

$$
\mathrm{Flt}_{d}^{\mathbb{R}}(X) \leq \frac{1}{n} \cdot \mathrm{Flt}_{d}(n \cdot X) \leq \frac{n+\lambda}{n} \cdot \mathrm{Flt}_{d}^{\mathbb{R}}(X)
$$

Hence, in order to determine the order of $\mathrm{Flt}_{d}(n \cdot X)$ as $n \rightarrow \infty$, it suffices to determine $\mathrm{Flt}_{d}^{\mathbb{R}}(X)$.

\subsection{Open questions}

Question 1. What is the asymptotical order of $\operatorname{ILC}(d)$ ?

Question 2. Is there an efficient algorithm to find $\operatorname{ILC}(P)$ (resp. $\operatorname{ILR}(P))$ for a given lattice polytope $P$ ?

We refer to [2, Theorem 6] for an answer when the number of lattice points in $P$ is fixed.

Question 3. What is the maximal width of a non-spanning lattice polytope in dimension d? 
Note that for $d=3$, Corollary 2.7 implies that the width of a non-spanning lattice polytope is at most 23 by using the bound from [3]. Compare this to the sharp bound of 3 .

Question 4. Is there a constant $N(d)$ such that any d-dimensional Delzant lattice polytope with width at least $N(d)$ is IDP?

For this, let us recall that a polytope is Delzant if all its normal cones are unimodular. Question 4 is a weaker version of Oda's conjecture, which asks whether all Delzant lattice polytopes are IDP. Note also Gubeladze's work [23] where he proved that lattice polytopes with sufficiently long edges are IDP. Theorem 2.10 shows that this cannot be generalized to lattice polytopes of sufficiently large width.

Question 5. Is there an infinite family of d-dimensional lattice polytopes with arbitrarily large widths that all have a non-unimodal $h^{*}$-vector?

This question is motivated by another main conjecture in this field, namely the question whether IDP implies unimodality of the $h^{*}$-vector (we refer to [41]). It is generally expected that lattice polytopes with sufficiently long edges have a unimodal $h^{*}$-vector.

Question 6. What is the order of $\mathrm{Flt}_{d}\left(n \cdot \Delta_{d}\right)$ ?

Question 7. What are the orders of $\mathrm{Flt}_{d}^{\mathbb{R}}\left(\Delta_{d}\right)$ and $\mathrm{Flt}_{d}^{\mathbb{R}}\left(\diamond_{d}\right)$, respectively?

Here, we denote the $d$-dimensional standard crosspolytope by

$$
\nabla_{d}:=\operatorname{conv}\left( \pm \mathbf{e}_{1}, \ldots, \pm \mathbf{e}_{d}\right) .
$$

This question is of interest in order to find lower bounds on the Gromov width (see Theorems 3.1 and 3.4, where one should recall that $\mathrm{Flt}_{d}^{\mathbb{R}}(n \cdot X)=n \cdot \mathrm{Flt}_{d}^{\mathbb{R}}(X)$ ).

\section{The relation of the lattice width to the Gromov width of symplectic manifolds}

\subsection{Background and notation}

The Gromov width of a $2 d$-dimensional symplectic manifold $(M, \omega)$ is defined as the supremum of the set of capacities $\pi r^{2}$ of balls of radii $r$ that can be symplectically embedded in $(M, \omega)$ (see [21]). We follow the convention in [32,33] and use the identification $S^{1}=\mathbb{R} / \mathbb{Z}$. There is a large interest in finding lower and upper bounds for the Gromov width, see e.g. [5,19,29,31-35,39]. One should remark that even for symplectic toric manifolds it is not known how to read off the Gromov width from the moment polytope. Here, we observe how closely the Gromov width and the lattice width of the moment polytope are related.

Let $X$ be a complex projective manifold of dimension $d, L$ an ample line bundle on $X$ and $\omega$ a Kähler form on $X$ representing the Chern class $c_{1}(L)$. In [29, Section 4], Kaveh constructs $\mathbb{Z}^{d}$-valued valuations on the field of rational functions $\mathbb{C}(X)$ to obtain associated Newton-Okounkov bodies $\Delta \subseteq \mathbb{R}^{d}$, i.e., $d$-dimensional convex bodies. We refer to [29] for the precise definition and choices involved. In the toric case, $\Delta$ is a Delzant polytope, i.e., a polytope whose normal fan consists of unimodular cones. 


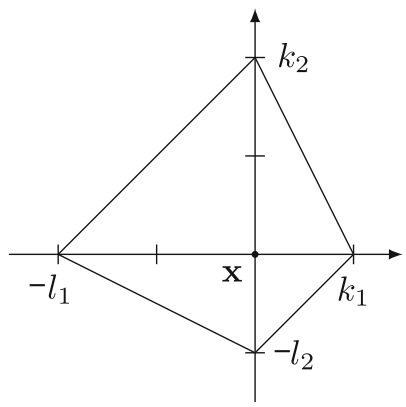

Fig. 1. A 2-dimensional diamond of size 3 with $\mathbf{x}=\mathbf{0}$

\subsection{Lower bounds on the Gromov width of symplectic manifolds}

The following result (see [29, Corollary 11.4]) is closely related to similar results in $[19,32,33]$.

Theorem 3.1. ([29, Corollary 11.4]) If $\Delta$ contains an $\mathbb{R}$-unimodular copy of $R \cdot \Delta_{d}$ (for $R>0$ ), then the Gromov width of $(X, \omega)$ is at least $R$.

Note that under the assumption of the theorem, we also have width $(\Delta) \geq R$. As an immediate application of Corollary 2.2, we see that the Gromov width of a symplectic manifold and the lattice width of its moment body are related.

Corollary 3.2. The Gromov width of $(X, \omega)$ is bounded from below by $\frac{\operatorname{width}(\Delta)}{\operatorname{Flt}(d) d}$.

In particular, for $d=2$, we get that $0.232 \cdot$ width $(\Delta)$ is a lower bound on the Gromov width. ${ }^{3}$ This bound is surely not sharp. We note that the lower bound in Corollary 3.2 is monotone with respect to inclusion of $\Delta$, a property that is conjectured to hold also for the Gromov width.

As we will need it later, let us describe a more general construction which has been used to give lower bounds for the Gromov width.

Definition 3.3. Let $\mathbf{b}_{1}, \ldots, \mathbf{b}_{d}$ be a lattice basis of $\mathbb{Z}^{d}, \mathbf{x} \in \mathbb{R}^{d}, a \in \mathbb{Z}_{\geq 1}$, and $k_{1}, l_{1}, \ldots, k_{d}, l_{d} \in \mathbb{R}_{\geq 0}$ with $k_{1}+l_{1}=a, \ldots, k_{d}+l_{d}=a$. Then

$$
\mathbf{x}+\operatorname{conv}\left(k_{1} \mathbf{b}_{1},-l_{1} \mathbf{b}_{1}, \ldots, k_{d} \mathbf{b}_{d},-l_{d} \mathbf{b}_{d}\right)
$$

will be called a diamond of size $a$. Note that $k_{1}=\cdots=k_{d}=1$ and $l_{1}=\cdots=$ $l_{d}=0$ yields a unimodular simplex, while for $k_{1}=l_{1}=1, \ldots, k_{d}=l_{d}=1$ we get the standard crosspolytope. See Fig. 1 for an illustration.

The following result generalizes Theorem 3.1. It is strictly speaking only proven in the toric situation, see [32,35,39], however, the proof of [29, Corollary 11.4] should carry through to the general case.

3 It follows from a recent result on Seshadri constants by Ambro and Ito [4, Theorem 0.1] that Flt $(d)$ can even be omitted in Corollary 3.2, hence, $0.5 \cdot$ width $(\Delta)$ would be a (sharp?) lower bound in dimension two. 
Theorem 3.4. If $\Delta$ contains an $\mathbb{R}$-unimodular copy of a diamond of size $R$ (for $R>0)$, then the Gromov width of $(X, \omega)$ is at least $R$.

Again, in this case we also have width $(\Delta) \geq R$. We refer to [26], in particular Example 5.4 therein, for the question how sharp such lower bound constructions can be.

\subsection{A conjectural upper bound on the Gromov width of symplectic manifolds}

The only nontrivial upper bound on the Gromov width of a symplectic toric manifold known to the authors was given by $\mathrm{Lu}$ (see [33]). For this, let us recall the following two definitions from [33].

Definition 3.5. Let $\Delta$ be a Delzant polytope with primitive inner facet normals $\mathbf{u}_{k} \in\left(\mathbb{R}^{d}\right)^{*}$ and facets $\left\{\mathbf{x} \in \Delta: \mathbf{u}_{k}(\mathbf{x})=-\phi_{k}\right\}$ for $\phi_{k} \in \mathbb{R}$. We define two numbers:

- $\Lambda(\Delta)$ is defined as the maximum over positive finite sums of the form $\sum_{k=1}^{m} a_{k} \phi_{k}$, where $a_{k}$ are nonnegative integers such that $\sum_{k=1}^{m} a_{k} \mathbf{u}_{k}=\mathbf{0}$, and $\sum_{k=1}^{m} a_{k} \leq d+1$.

- $\Upsilon(\Delta)$ is defined as the minimum over positive finite sums of the form $\sum_{k=1}^{m} a_{k} \phi_{k}$, where $a_{k}$ are nonnegative integers such that $\sum_{k=1}^{m} a_{k} \mathbf{u}_{k}=\mathbf{0}$.

Remark 3.6. Note that $\Lambda(\Delta)$ is a well-defined finite number (cf. [33] or [8, Prop. 3.2]). Furthermore, it is straightforward to show that both $\Lambda(\Delta)$ and $\Upsilon(\Delta)$ are invariant under translations by real vectors. After an appropriate translation, we may assume that the origin is in the interior of the polytope $\Delta$, and thus all $\phi_{k}$ are positive. From this it straightforwardly follows that a finite sum $\sum_{k=1}^{m} a_{k} \phi_{k}$ as in Definition 3.5 is positive if and only if at least one $a_{k}$ is positive. Furthermore, note that in general $\Upsilon(\Delta) \leq \Lambda(\Delta)$.

Lu proves the following two results.

Theorem 3.7. ([33, Theorem 1.1]) The Gromov width of $(X, \omega)$ is bounded from above by $\Lambda(\Delta)$.

Theorem 3.8. ([33, Theorem 1.2], see also [26, Theorem 5.5]) If $X$ is a toric Fano manifold, then the Gromov width of $(X, \omega)$ is bounded from above by $\Upsilon(\Delta)$.

We observe that Lu's sharper upper bound $\Upsilon(\Delta)$ is simply the lattice width.

Proposition 3.9. If $\Delta$ is a Delzant polytope, then $\Upsilon(\Delta)=\operatorname{width}(\Delta)$.

The proof follows by combining the next two lemmata. For this, let us define the facet width of a polytope $\Delta \subseteq \mathbb{R}^{d}$ as the minimum of width $(\Delta)$ where $\mathbf{u} \in\left(\mathbb{Z}^{d}\right)^{*}$ ranges over all facet normals of $\Delta$.

Lemma 3.10. Let $\Delta \subseteq \mathbb{R}^{d}$ be a Delzant polytope. Then $\Upsilon(\Delta)$ equals the facet width of $\Delta$. 
Proof. Let $a_{k}, \mathbf{u}_{k}, \phi_{k}$ be given as in the definition of $\Upsilon(\Delta)$ (see Definition 3.5).

We first show that $\Upsilon(\Delta)$ is bounded from below by the facet width of $\Delta$. For this, it suffices to show that, for any positive finite sum $\sum_{k=1}^{m} a_{k} \phi_{k}$ as in Definition 3.5, there is an inner facet normal $\mathbf{u}$ of $\Delta \operatorname{such}$ that $\operatorname{width}_{\mathbf{u}}(\Delta) \leq \sum_{k=1}^{m} a_{k} \phi_{k}$. Suppose $a_{1} \neq 0$ and define $b_{1}:=a_{1}-1, b_{k}:=a_{k}$ for $k>1$. Hence, $\mathbf{u}_{1}+\sum_{k=1}^{m} b_{k} \mathbf{u}_{k}=\mathbf{0}$. For $\mathbf{x} \in \Delta$, we have $\mathbf{u}_{1}(\mathbf{x}) \geq-\phi_{1}$ and $-\mathbf{u}_{1}(\mathbf{x})=\sum_{k=1}^{m} b_{k} \mathbf{u}_{k}(\mathbf{x}) \geq-\sum_{k=1}^{m} b_{k} \phi_{k}$, so that $\mathbf{u}_{1}(\mathbf{x}) \leq \sum_{k=1}^{m} b_{k} \phi_{k}$. Thus, width $\mathbf{u}_{1}(\Delta) \leq \phi_{1}+\sum_{k=1}^{m} b_{k} \phi_{k}=\sum_{k=1}^{m} a_{k} \phi_{k}$.

For the reverse inequality, suppose $\mathbf{u}_{m} \in\left(\mathbb{Z}^{d}\right)^{*}$ is a primitive inner facet normal of $\Delta$ for which the facet width is attained, i.e., width $\mathbf{u}_{m}(\Delta)=$ facet width of $\Delta$. It suffices to show that there is a positive finite sum $\sum_{k=1}^{m} a_{k} \phi_{k}$ as in Definition 3.5 which bounds width $\mathbf{u}_{m}(\Delta)$ from below. Note that both the facet width and $\Upsilon(\Delta)$ are invariant under translations by real vectors. Hence, we may assume that $\mathbf{0}$ is an element of the facet corresponding to $\mathbf{u}_{m}$, and thus, $\phi_{m}=0$. There exist primitive inner facet normals, say $\mathbf{u}_{1}, \ldots, \mathbf{u}_{d}$ (up to reordering the rays), that span a unimodular cone $\sigma$ of the inner normal fan of $\Delta$ such that $-\mathbf{u}_{m}=\sum_{k=1}^{d} a_{k} \mathbf{u}_{k}$ with $a_{k} \in \mathbb{Z}_{\geq 0}$. Let the remaining $a_{k}$ vanish, i.e., $a_{d+1}=\cdots=a_{m}=0$, and let $\mathbf{x}$ be the vertex of $\Delta$ corresponding to $\sigma$. In particular, $\mathbf{u}_{k}(\mathbf{x})=-\phi_{k}$ for $k=1, \ldots, d$. Then $\sum_{k=1}^{m} a_{k} \phi_{k}=-\sum_{k=1}^{d} a_{k} \mathbf{u}_{k}(\mathbf{x})=\mathbf{u}_{m}(\mathbf{x}) \leq \operatorname{width}_{\mathbf{u}_{m}}(\Delta)$.

It remains to observe the following.

Lemma 3.11. The lattice width of a Delzant polytope coincides with its facet width.

Proof. Let $\Delta \subseteq \mathbb{R}^{d}$ be a Delzant polytope. Clearly, the lattice width of $\Delta$ is less than or equal to the facet width of $\Delta$.

For the reverse inequality, let $\mathbf{u} \in\left(\mathbb{Z}^{d}\right)^{*}$ with width $\mathbf{u}(\Delta)=\operatorname{width}(\Delta)$. As $\Delta$ is a Delzant polytope, we can replace $\Delta$ by a unimodular copy such that $\mathbf{u}=\sum_{i=1}^{d} k_{i} \mathbf{e}_{i}$ for $k_{i} \in \mathbb{Z}_{\geq 0}$, where the standard basis $\mathbf{e}_{1}, \ldots, \mathbf{e}_{d}$ of $\mathbb{Z}^{d}$ spans a cone in the inner normal fan of $\Delta$. Suppose $k_{1} \geq 1$ and let $\mathbf{v}$ be the vertex of $\Delta$ corresponding to the cone spanned by $\mathbf{e}_{1}, \ldots, \mathbf{e}_{d}$. By translating by a real vector we may assume that $\mathbf{v}=\mathbf{0}$. Let $\mathbf{w}_{1}$ be a vertex of $\Delta$ maximizing $\mathbf{e}_{1}$ on $\Delta$, so that width $\mathbf{e}_{1}(\Delta)=\mathbf{e}_{1}\left(\mathbf{w}_{1}\right)$. We have

$$
\mathbf{u}\left(\mathbf{w}_{1}\right) \leq \operatorname{width}_{\mathbf{u}}(\Delta)=\operatorname{width}(\Delta) \leq \text { width }_{\mathbf{e}_{1}}(\Delta)=\mathbf{e}_{1}\left(\mathbf{w}_{1}\right) .
$$

Hence, $0 \geq\left(\mathbf{u}-\mathbf{e}_{1}\right)\left(\mathbf{w}_{1}\right)=\left(\left(k_{1}-1\right) \mathbf{e}_{1}+\sum_{k=2}^{d} k_{i} \mathbf{e}_{i}\right)\left(\mathbf{w}_{1}\right) \geq 0$, i.e., $\mathbf{u}\left(\mathbf{w}_{1}\right)=$ $\mathbf{e}_{1}\left(\mathbf{w}_{1}\right)$. The statement follows.

This motivates the question whether Lu's sharper upper bound also holds in general.

Conjecture 3.12. ${ }^{4}$ The Gromov width of $(X, \omega)$ is bounded from above by the lattice width of $\Delta$.

This is also formulated as a question in [26, Question 5.10].

\footnotetext{
4 A proof of Conjecture 3.12 in dimension 2 has been subsequently found by Chaidez and Wormleighton [17]. It also follows from [16, Prop. 2.8] that Conjecture 3.12 holds for a large class of Delzant polytopes that includes graph associahedra.
} 
Remark 3.13. Here is a heuristical argument in favor of this conjecture. Put a unimodular copy $\Delta^{\prime}$ of $\Delta$ between two parallel coordinate hyperplanes whose distance equals the lattice width. Then clearly $\Delta^{\prime}$ is included in a large rectangular box of the same lattice width. It is expected (but wide open) that the Gromov width should respect inclusion of the moment polytopes. Therefore, the Gromov width should be at most as large as that of the symplectic toric manifold corresponding to the rectangular box, i.e., a product of projective lines. However, in this case the Gromov width is known (e.g., by Gromov's proof of the non-squeezing theorem) and equals the smallest size of an edge, which is the lattice width of $\Delta$.

The reader should be aware that the Gromov width may differ from the lattice width as is shown in [26, Example 5.6]. We remark that it seems not to be even known whether fixing the lattice width of $\Delta$ imposes any bounds on the Gromov width.

Remark 3.14. Lu claims in [33, Remark 1.5] that his upper bound $\Upsilon(\Delta)$ (which by above results equals the lattice width of $\Delta$ ) does not hold in the non-Fano case by exhibiting an explicit example of a polygon space. This would contradict Conjecture 3.12 and the argument in Remark 3.13 would show that the Gromov width were not monotone with respect to inclusions. However, we couldn't verify his claim, his computations seem to be wrong. In fact, we get $\Upsilon(\Delta)=2$ while Lu claims $\Upsilon(\Delta)=\frac{1}{6}$. In particular, $\Upsilon(\Delta)=2$ coincides with the Gromov width by [35, Theorem 1].

Remark 3.15. The reader may wonder why we expressed Conjecture 3.12 in terms of the lattice width instead of the facet width. This was to make the relation to the other results in this paper more apparent and to stress the fact that while the lattice width of convex bodies is monotone with respect to inclusion (as is also conjectured for the Gromov width), this is not true for the facet width of (nonDelzant) polytopes. For instance, for a natural number $k \geq 1$, the lattice triangle with vertices $(0,0),(k,-1)$, and $(k+1,1)$ is contained in the lattice rectangle $[0, k+1] \times[-1,1]$. While the rectangle has facet width 2 , the facet width of the triangle is linearly increasing in $k$ (indeed it equals $2 k+1$ ).

\subsection{Dimension 2}

As in dimension 2 any smooth complete toric surface is obtained from $\mathbb{P}^{2}, \mathbb{P}^{1} \times \mathbb{P}^{1}$ or a Hirzebruch surface $\mathcal{H}_{a}$ (for $a \in \mathbb{Z}_{\geq 1}$ ) by a sequence of blows-ups at toric fixed points (e.g., see [20, page 43]), a proof ${ }^{5}$ of the 2-dimensional case of Conjecture 3.12 could be achieved by an appropriate generalization of [33, Theorem 6.2], where Lu studies how the upper bound $\Upsilon(\Delta)$ behaves under blow-ups of toric Fano manifolds at toric fixed points.

Let us note the folklore fact that for these minimal toric surfaces the Gromov width indeed equals the lattice width.

5 The proof of Conjecture 3.12 in dimension 2 by Chaidez and Wormleighton [17] showed the stronger monotonicity property and then followed the heuristics in Remark 3.13. 
Lemma 3.16. If $X$ is $\mathbb{P}^{2}, \mathbb{P}^{1} \times \mathbb{P}^{1}$ or $\mathcal{H}_{a}$, then the Gromov width of $(X, \omega)$ equals the lattice width of $\Delta$.

Proof. The cases $X=\mathbb{P}^{2}$ and $X=\mathbb{P}^{1} \times \mathbb{P}^{1}$ are straightforwardly verified. It remains to check the case of Hirzebruch surfaces.

We may assume that $\Delta$ is a 4-gon with vertices $\left(\begin{array}{l}0 \\ 0\end{array}\right),\left(\begin{array}{l}x \\ 0\end{array}\right),\left(\begin{array}{l}0 \\ y\end{array}\right),\left(\begin{array}{c}x \\ y-a x\end{array}\right)$ with $y>a x>0$. As $y>x$, the facet width (hence, the lattice width by Lemma 3.11) of $\Delta$ equals $x$. As $\Delta$ contains $x \Delta_{2}$, Theorem 3.1 implies that the Gromov width is at least $x$. For the reverse inequality, we distinguish two cases. If $a=1$, then $X$ is Fano, hence, the statement follows by Theorem 3.8 and Proposition 3.9 (this was also proven in [39, p. 206]). If $a>1$, we use Theorem 3.7 to bound the Gromov width from above. Indeed, the only nontrivial linear combination (with nonnegative integer coefficients) of ray generators in the inner normal fan of $\Delta$ that sum up to $\left(\begin{array}{l}0 \\ 0\end{array}\right)$ and have at most three summands, is $\left(\begin{array}{c}-1 \\ 0\end{array}\right)+\left(\begin{array}{l}1 \\ 0\end{array}\right)=\left(\begin{array}{l}0 \\ 0\end{array}\right)$, and thus, $\Lambda(\Delta)=x$ by Definition 3.5 .

We couldn't find the following observation on toric surfaces of small Gromov width in the literature.

Proposition 3.17. If $(X, \omega)$ is a symplectic toric surface whose moment polytope $\Delta$ is a Delzant lattice polygon, then its Gromov width equals 1 if and only if the lattice width of $\Delta$ equals 1 . In this case, $X \cong \mathbb{P}^{2}, X \cong \mathbb{P}^{1} \times \mathbb{P}^{1}$ or $X$ is a Hirzebruch surface.

Proof. If $\Delta$ has no interior lattice points, then it is well known (e.g., see [38, Theorem 2]) that there are two cases. Either, $\Delta \cong 2 \Delta_{2}$, so lattice width and Gromov width are both 2 (e.g. by Theorems 3.1 and 3.8 together with Proposition 3.9). Or, $\Delta \cong \operatorname{conv}\left(\left(\begin{array}{l}0 \\ 0\end{array}\right),\left(\begin{array}{l}1 \\ 0\end{array}\right),\left(\begin{array}{l}0 \\ y\end{array}\right),\left(\begin{array}{c}1 \\ y-a\end{array}\right)\right)$ with $y \geq a \geq 0$, i.e., $X \cong \mathbb{P}^{2}$, $X \cong \mathbb{P}^{1} \times \mathbb{P}^{1}$, or $X$ is a Hirzebruch surface (note that we only consider the case where $\Delta$ is a smooth polygon). Hence, the lattice width and the Gromov width both equal 1 (see Lemma 3.16).

Suppose that $\Delta$ has an interior lattice point. We may assume that $\mathbf{0}$ is a vertex of $\Delta$ with edge directions $\mathbf{e}_{1}, \mathbf{e}_{2}$. As $\Delta$ is a Delzant lattice polygon with interior lattice points, convexity implies that $\left(\begin{array}{l}1 \\ 1\end{array}\right) \in \Delta$. Moreover, it is easy to see that $\left(\begin{array}{l}1 \\ 1\end{array}\right)$ cannot be on the boundary, so it is a lattice point in the interior of $\Delta$. Consider the edge with vertex $\mathbf{0}$ and edge direction $\mathbf{e}_{1}$. Let $\mathbf{w}_{1}$ be the other vertex on that edge. As $\Delta$ is a Delzant lattice polygon, there exists a lattice point $\mathbf{w}_{1}^{\prime}$ of $\Delta$ that lies in a common edge with $\mathbf{w}_{1}$ and has second coordinate 1 . By convexity and as $\left(\begin{array}{l}1 \\ 1\end{array}\right)$ is not on the boundary of $\Delta$, this implies that $(2,1) \in \Delta$. In the same way, one proves that $(1,2) \in \Delta$. This shows that the diamond conv $\left(\left(\begin{array}{l}1 \\ 0\end{array}\right),\left(\begin{array}{l}1 \\ 2\end{array}\right),\left(\begin{array}{l}2 \\ 1\end{array}\right),\left(\begin{array}{l}0 \\ 1\end{array}\right)\right)$ of size 2 is in $\Delta$, and thus the Gromov width is at least 2 by Theorem 3.4. 


\section{Proof of Theorem 2.1}

The proof uses general results on successive minima and covering minima to reduce the problem to translates of parallelepipeds.

Let us recall the following standard notions in discrete geometry. The Minkowski sum of two subsets $A, B \subseteq \mathbb{R}^{d}$ is given by $A+B=\{a+b: a \in A, b \in B\}$ and it can be recursively extended to finite families $A_{1}, \ldots, A_{k} \subseteq \mathbb{R}^{d}$ in which case we write $\sum_{i=1}^{k} A_{i}$. For a convex body $K \subseteq \mathbb{R}^{d}$, the $d$-th successive minimum of its difference body $K-K=\{x-y: x, y \in K\}$ is defined as follows:

$$
\lambda_{d}(K-K):=\inf \left\{\lambda>0: \operatorname{dim}\left(\operatorname{span}\left\{\lambda(K-K) \cap \mathbb{Z}^{d}\right\}\right)=d\right\},
$$

while the definition of the $d$-th covering minimum of $K$ is given by:

$$
\mu_{d}(K):=\inf \left\{\mu>0: \mu K+\mathbb{Z}^{d}=\mathbb{R}^{d}\right\} .
$$

Lemma 4.1. Let $K \subseteq \mathbb{R}^{d}$ be a convex body. Then we have $\lambda_{d}(K-K) \leq \frac{\operatorname{Flt}(d)}{\operatorname{width}(K)}$.

Proof. We set $\tau:=\frac{\text { Flt }(d)}{\operatorname{width}(K)}$. By [30, Lemma 2.4], $\lambda_{d}(K-K) \leq \mu_{d}(K)$. It suffices to show $\mu_{d}(K) \leq \tau$. For this, let $\mathbf{x} \in \mathbb{R}^{d}$ and set $K^{\prime}:=\tau K-\mathbf{x}$. As width $\left(K^{\prime}\right)=\operatorname{Flt}(d)$, there is a lattice point $\mathbf{y}$ in $K^{\prime}$, i.e. $\mathbf{y}=\mathbf{z}-\mathbf{x}$ for some $\mathbf{z} \in \tau K$. Thus $\mathbf{x} \in \tau K+\mathbb{Z}^{d}$.

Given two points $\mathbf{v}, \mathbf{w} \in \mathbb{R}^{d}$, we write $[\mathbf{v}, \mathbf{w}]$ for the line segment between those two points, i.e., $[\mathbf{v}, \mathbf{w}]=\operatorname{conv}(\mathbf{v}, \mathbf{w})$.

The following result is folklore for lattice parallelepipeds.

Lemma 4.2. Let $\mathbf{v}_{1}, \ldots, \mathbf{v}_{d} \in \mathbb{Z}^{d}$ be linearly independent. If $\mathbf{a} \in \mathbb{Z}^{d}$ (resp. $\mathbf{a} \in$ $\mathbb{R}^{d}$ ), then the lattice parallelepiped $P:=\mathbf{a}+\sum_{i=1}^{d}\left[\mathbf{0}, \mathbf{v}_{i}\right]$ (resp. the parallelepiped $\left.P:=\mathbf{a}+\sum_{i=1}^{d}\left[\mathbf{0}, 2 \mathbf{v}_{i}\right]\right)$ contains a unimodular copy of the standard simplex $\Delta_{d}$.

Note the stronger assumptions in the case of real translates of lattice parallelepipeds.

Proof. We proceed by induction on the dimension $d$. The crucial difference in the argument is evident in dimension $d=1$ : If $\mathbf{a} \in \mathbb{Z}^{d}$, then it suffices to observe that any interval with integral endpoints contains two consecutive integer points. However, if $\mathbf{a} \in \mathbb{R}^{d}$, then one needs to assume that $P$ is a segment of length at least 2 to ensure that $P$ contains two consecutive integer points.

Now, let $d \geq 2$. By applying an appropriate (linear) unimodular transformation, we may assume $\mathbf{v}_{1}, \ldots, \mathbf{v}_{d-1} \in \mathbb{Z}^{d-1} \times\{\boldsymbol{0}\}$. Consider the projection $\pi: \mathbb{R}^{d} \rightarrow \mathbb{R}$ onto the last coordinate, i.e., given by $\pi\left(x_{1}, \ldots, x_{d}\right)=x_{d}$. Now, we distinguish the two cases.

If $\mathbf{a} \in \mathbb{Z}^{d}$, then we apply the induction hypothesis to $P^{\prime}:=\mathbf{a}+$ $\sum_{i=1}^{d-1}\left[\mathbf{0}, \mathbf{v}_{i}\right] \subseteq \mathbb{R}^{d-1} \times\{\pi(\mathbf{a})\}$ and deduce in $P^{\prime}$ the existence of a unimodular copy conv $\left(\mathbf{p}_{0}^{\prime}, \ldots, \mathbf{p}_{d-1}^{\prime}\right)$ of $\Delta_{d-1}$. Next, we observe that the intersection of $P$ with $\mathbb{R}^{d-1} \times\{\pi(\mathbf{a})+1\}$ is a translation of the lattice parallelepiped $\left(\sum_{i=1}^{d-1}\left[\mathbf{0}, \mathbf{v}_{i}\right]\right) \times$ 
$\{\pi(\mathbf{a})+1\}$. Hence, it must contain a lattice point $\mathbf{p}^{\prime \prime} \in \mathbb{Z}^{d-1} \times\{\pi(\mathbf{a})+1\}$. Therefore, $\operatorname{conv}\left(\mathbf{p}_{0}^{\prime}, \ldots, \mathbf{p}_{d-1}^{\prime}, \mathbf{p}^{\prime \prime}\right)$ is a unimodular copy of $\Delta_{d}$ contained in $P$.

Let $\mathbf{a} \notin \mathbb{Z}^{d}$. We have $\pi(P)=\pi(\mathbf{a})+\left[\mathbf{0}, 2 \pi\left(\mathbf{v}_{d}\right)\right]$ with $0 \neq \pi\left(\mathbf{v}_{d}\right) \in \mathbb{Z}$. As $\pi(P)$ is a segment, it follows by the base case of the induction that it contains two consecutive integers, say $k$ and $k+1$. Thus (as $\mathbf{v}_{1}, \ldots, \mathbf{v}_{d-1}$ have last coordinate 0 ) there are $\mathbf{v}^{\prime}, \mathbf{v}^{\prime \prime} \in\left[\mathbf{0}, 2 \mathbf{v}_{d}\right]$ such that $\pi\left(\mathbf{a}+\mathbf{v}^{\prime}\right)=k$ and $\pi\left(\mathbf{a}+\mathbf{v}^{\prime \prime}\right)=k+1$. We note that $\mathbf{a}+\mathbf{v}^{\prime}, \mathbf{a}+\mathbf{v}^{\prime \prime}$ need not to be lattice points. We apply the induction hypothesis to $P^{\prime}:=\mathbf{a}+\mathbf{v}^{\prime}+\sum_{i=1}^{d-1}\left[\mathbf{0}, 2 \mathbf{v}_{i}\right] \subseteq \mathbb{R}^{d-1} \times\{k\}$ and deduce in $P^{\prime}$ the existence of a unimodular copy $\operatorname{conv}\left(\mathbf{p}_{0}^{\prime}, \ldots, \mathbf{p}_{d-1}^{\prime}\right)$ of $\Delta_{d-1}$. Analogously, the induction hypothesis yields that the set $P^{\prime \prime}:=\mathbf{a}+\mathbf{v}^{\prime \prime}+\sum_{i=1}^{d-1}\left[\mathbf{0}, 2 \mathbf{v}_{i}\right] \subseteq \mathbb{R}^{d-1} \times\{k+1\}$ also contains a unimodular copy $\operatorname{conv}\left(\mathbf{p}_{0}^{\prime \prime}, \ldots, \mathbf{p}_{d-1}^{\prime \prime}\right)$ of $\Delta_{d-1}$. By construction, for any $i \in\{0, \ldots, d-1\}$ the convex hull of the vectors $\mathbf{p}_{0}^{\prime}, \ldots, \mathbf{p}_{d-1}^{\prime}, \mathbf{p}_{i}^{\prime \prime}$ form a unimodular copy of $\Delta_{d}$ contained in $P$.

Clearly, the previous proof implies more than what we need. For instance, it can be easily modified to show that any such parallelepiped $P$ has at least $2^{d}$ many lattice points. However, the reader is cautioned not to jump to the conclusion that it proves the existence of a unimodular copy of the unit cube $[0,1]^{d}$ in $P$.

Proof of Theorem 2.1. (1) By Lemma 4.1, $\lambda_{d}(K-K) \leq \frac{1}{2 d}$, i.e., there exist $d$ segments $I_{i}=\left[\mathbf{a}_{i}, \mathbf{b}_{i}\right]$ with $i \in\{1, \ldots, d\}$ contained in $\frac{1}{2 d} K$ such that the $d$ vectors $\mathbf{v}_{i}:=\mathbf{b}_{i}-\mathbf{a}_{i}$ are linearly independent and belong to $\mathbb{Z}^{d}$. This implies $I_{1}+\cdots+I_{d} \subseteq \frac{1}{2} K$. Hence,

$$
2\left(I_{1}+\cdots+I_{d}\right) \subseteq K .
$$

The latter inclusion can be written as

$$
\mathbf{a}+\sum_{i=1}^{d}\left[\mathbf{0}, 2 \mathbf{v}_{i}\right] \subseteq K
$$

with $\mathbf{a}:=2\left(\mathbf{a}_{1}+\cdots+\mathbf{a}_{d}\right)$. The statement follows from Lemma 4.2.

(2) Again by Lemma 4.1, $\lambda_{d}(K-K) \leq \frac{1}{d}$, i.e., there exist $d$ segments $I_{i}=\left[\mathbf{a}_{i}, \mathbf{b}_{i}\right]$ with $i \in\{1, \ldots, d\}$ contained in $\frac{1}{d} K$ such that the $d$ vectors $\mathbf{v}_{i}:=\mathbf{b}_{i}-\mathbf{a}_{i}$ are linearly independent and belong to $\mathbb{Z}^{d}$. This implies $I_{1}+\cdots+I_{d} \subseteq K$. Hence,

$$
\mathbf{a}+\sum_{i=1}^{d}\left[\mathbf{0}, \mathbf{v}_{i}\right] \subseteq K
$$

with $\mathbf{a}:=\mathbf{a}_{1}+\cdots+\mathbf{a}_{d}$. The statement follows from Lemma 4.2.

\section{Proof of Theorem 2.10}

For given $k \in \mathbb{N}$ with $k \geq 3$, we define the three-dimensional lattice polytope

$$
P:=\operatorname{conv}\left(\{(3,0,-1),(0,2,-1)\} \cup[0, k]^{3}\right) \quad \text { (see Fig. 2). }
$$




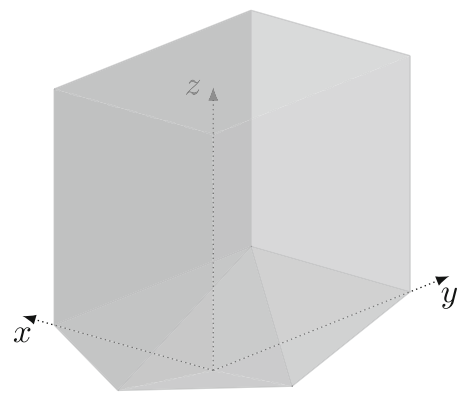

Fig. 2. The polytope $P$ for $k=5$ (as seen from below the $x-y$-plane)

We will show that $P$ has the desired property. Then for $d>3$, one simply takes the Cartesian product of $P$ with $[0, k]^{d-3}$. Let us first observe that $P$ has lattice width $k$, as the width is at most $k$ in the direction $(1,0,0)$, while on the other hand it has to be at least $k$, because $P$ contains $[0, k]^{3}$.

Let $t \in \mathbb{N}$ with $t \geq 2$. Let us abbreviate $Z_{1}:=\mathbb{Z}^{3} \cap P$, and

$$
Z_{t}:=\underbrace{Z_{1}+\cdots+Z_{1}}_{t \text { times }} .
$$

We will show that the lattice point

$$
p:=(3 t-4,1,1-t)
$$

satisfies $p \in \operatorname{conv}\left(Z_{t}\right)=t P$ but $p \notin Z_{t}$.

For this, we consider

$$
Z_{t}^{\prime}:=\left\{\mathbf{x} \in \mathbb{Z}^{2}:(\mathbf{x}, 1-t) \in Z_{t}\right\} .
$$

In order to determine $Z_{t}^{\prime}$, we observe that the last coordinates of the points in $Z_{1}$ comprise the set $\{-1,0, \ldots, k\}$. We thus need to determine the possibilities for values $s_{1}, \ldots, s_{t} \in\{-1,0, \ldots, k\}$ such that the sum $s_{1}+\cdots+s_{t}$ is equal to $1-t$. It is easily seen that one of the values $s_{1}, \ldots, s_{t}$ has to be equal to 0 and the remaining ones to -1 for the latter to be fulfilled. Since $(3,0,-1)$ and $(0,2,-1)$ are the only two points in $Z_{1}$ with last component -1 and $\{0, \ldots, k\}^{2} \times\{0\}$ is the set of all points of $Z_{1}$ with last component 0 , we get

$$
Z_{t}^{\prime}=\underbrace{\{(3,0),(0,2)\}+\cdots+\{(3,0),(0,2)\}}_{(t-1) \text { times }}+\{0, \ldots, k\}^{2} .
$$

We observe that

$$
\begin{gathered}
(3 t-4,1)=\frac{1}{2}((t-1)(3,0)+(0,0))+\frac{1}{2} \\
((t-2)(3,0)+(0,2)+(1,0)) \in \operatorname{conv}\left(Z_{t}^{\prime}\right) .
\end{gathered}
$$

Thus, $p \in \operatorname{conv}\left(Z_{t}\right)$. 
Finally, we notice that $(3 t-4,1)=(3(t-1)-1,1) \notin Z_{t}^{\prime}$. Otherwise, it could be expressed as a sum as on the right side of (1). However, as the second component of $\{0, \ldots, k\}^{2}$ is nonnegative, no $(0,2)$ would be allowed as a summand, so it would be a sum of $(t-1)(3,0)$ and a point in $\{0, \ldots, k\}^{2}$, a contradiction. Therefore, $p \notin Z_{t}$.

Acknowledgements We are particularly grateful to Milena Pabiniak for introducing us to combinatorial problems regarding the Gromov width and patiently answering our questions. We would also like to thank Gabriele Balletti for useful discussions and computations regarding non-spanning lattice polytopes. We are thankful to the anonymous referee for several suggestions and corrections.

Funding The first and last authors are PIs in the Research Training Group Mathematical Complexity Reduction funded by the Deutsche Forschungsgemeinschaft (DFG, German Research Foundation) - 314838170, GRK 2297 MathCoRe. The last author was also partially supported by the Vetenskapsrådet grant NT:2014-3991 (as an affiliated researcher with Stockholm University). The second author is supported by a Nottingham Research Fellowship from the University of Nottingham.

Open Access This article is licensed under a Creative Commons Attribution 4.0 International License, which permits use, sharing, adaptation, distribution and reproduction in any medium or format, as long as you give appropriate credit to the original author(s) and the source, provide a link to the Creative Commons licence, and indicate if changes were made. The images or other third party material in this article are included in the article's Creative Commons licence, unless indicated otherwise in a credit line to the material. If material is not included in the article's Creative Commons licence and your intended use is not permitted by statutory regulation or exceeds the permitted use, you will need to obtain permission directly from the copyright holder. To view a copy of this licence, visit http://creativecommons.org/ licenses/by/4.0/.

\section{Declarations}

Conflict of interest The authors declare that they have no conflict of interest.

Funding Open Access funding enabled and organized by Projekt DEAL.

\section{References}

[1] Aliev, I., Averkov, G., Loera, J. A. De., Oertel, T.: Optimizing sparsity over lattices and semigroups. In: Integer Programming and Combinatorial Optimization, Lecture Notes in Computer Science, vol. 12125, Springer, Cham, pp. 40-51 (2020)

[2] Aliev, I., Averkov, G., Loera, J. A. De, Oertel, T.: Sparse representation of vectors in lattices and semigroups. In: Mathematical Programming (2021)

[3] Averkov, G., Codenotti, G., Macchia, A., Santos, F.: A local maximizer for lattice width of 3-dimensional hollow bodies (2019). arXiv preprint arXiv:1907.06199

[4] Ambro, F., Ito, A.: Successive minima of line bundles. Adv. Math. 365, 38 (2020)

[5] Arezzo, C., Loi, A., Zuddas, F.: Some remarks on the symplectic and Kähler geometry of toric varieties. Ann. Mat. Pura Appl. 195(4), 1287-1304 (2016) 
[6] Averkov, G.: On finitely generated closures in the theory of cutting planes. Discrete Optim. 9(4), 209-215 (2012)

[7] Barvinok, A.: A Course in Convexity, Graduate Studies in Mathematics, vol. 54. American Mathematical Society, Providence (2002)

[8] Batyrev, V.V.: On the classification of smooth projective toric varieties. Tohoku Math. J. 43(4), 569-585 (1991)

[9] Bruns, W., Gubeladze, J.: Polytopes, Rings, and $K$-theory. Springer Monographs in Mathematics, Springer, Dordrecht (2009)

[10] Bruns, W., Gubeladze, J., Henk, M., Martin, A., Weismantel, R.: A counterexample to an integer analogue of Carathéodory's theorem. J. Reine Angew. Math. 510, 179-185 (1999)

[11] Banaszczyk, W., Litvak, A.E., Pajor, A., Szarek, S.J.: The flatness theorem for nonsymmetric convex bodies via the local theory of Banach spaces. Math. Oper. Res. 24(3), 728-750 (1999)

[12] Beck, M., Robins, S.: Computing the continuous discretely. In: Undergraduate Texts in Mathematics, 2nd edn., Integer-Point Enumeration in Polyhedra, With Illustrations by David Austin, Springer, New York (2015)

[13] Blanco, M., Santos, F.: Enumeration of lattice 3-polytopes by their number of lattice points. Discrete Comput. Geom. 60(3), 756-800 (2018)

[14] Codenotti, G., Santos, F.: Hollow polytopes of large width. Proc. Am. Math. Soc. 148(2), 835-850 (2020)

[15] Codenotti, G., Santos, F.: Non-spanning lattice 3-polytopes. J. Combin. Theory Ser. A 161, 112-133 (2019)

[16] Choi, S., Hwang, T.: Gromov Width of Symplectic Toric Manifolds Associated With Graphs, vol. 09729 (2011)

[17] Chaidez, J., Wormleighton, B.: ECH embedding obstructions for rational surfaces (2020). arXiv preprint arXiv:2008.10125

[18] Eisenbrand, F., Shmonin, G.: Carathéodory bounds for integer cones. Oper. Res. Lett. 34(5), 564-568 (2006)

[19] Fang, X., Littelmann, P., Pabiniak, M.: Simplices in Newton-Okounkov bodies and the Gromov width of coadjoint orbits. Bull. Lond. Math. Soc. 50(2), 202-218 (2018)

[20] Fulton, W.: Introduction to toric varieties. In: Annals of Mathematics Studies, The William H. Roever Lectures in Geometry, vol. 131, Princeton University Press, Princeton (1993)

[21] Gromov, M.: Pseudo holomorphic curves in symplectic manifolds. Invent. Math. 82(2), 307-347 (1985)

[22] Gubeladze, J.: Normal polytopes. In: Proceedings of 22nd International Conference on Formal Power Series and Algebraic Combinatorics, San Francisco, pp. 4-8 (2010)

[23] Gubeladze, J.: Convex normality of rational polytopes with long edges. Adv. Math. 230(1), 372-389 (2012)

[24] Hofscheier, J., Katthän, L., Nill, B.: Spanning lattice polytopes and the uniform position principle (2017). arXiv preprint arXiv:1711.09512

[25] Hofscheier, J., Katthän, L., Nill, B.: Ehrhart theory of spanning lattice polytopes. Int. Math. Res. Not. IMRN 19, 5947-5973 (2018)

[26] Hwang, T., Lee, E., Suh, D. Y.: The Gromov width of generalized Bott manifolds. In: International Mathematics Research Notices, vol. rnz066 (2019)

[27] Hibi, T., Tsuchiya, A.: Classification of lattice polytopes with small volume (2017). arXiv preprint arXiv: 1708.00413

[28] Hurkens, C.A.J.: Blowing up convex sets in the plane. Linear Algebra Appl. 134, 121128 (1990) 
[29] Kaveh, K.: Toric degenerations and symplectic geometry of smooth projective varieties. J. Lond. Math. Soc. 99(2), 377-402 (2019)

[30] Kannan, R., Lovász, L.: Covering minima and lattice-point-free convex bodies. Ann. Math. 128(3), 577-602 (1988)

[31] Karshon, Y., Tolman, S.: The Gromov width of complex Grassmannians. Algebraic Geom. Topol. 5, 911-922 (2005)

[32] Latschev, J., McDuff, D., Schlenk, F.: The Gromov width of 4-dimensional tori. Geom. Topol. 17(5), 2813-2853 (2013)

[33] Lu, G.: Symplectic capacities of toric manifolds and related results. Nagoya Math. J. 181, 149-184 (2006)

[34] Marinković, A., Pabiniak, M.: Every symplectic toric orbifold is a centered reduction of a Cartesian product of weighted projective spaces. Int. Math. Res. Not. IMRN 23, 12432-12458 (2015)

[35] Mandini, A., Pabiniak, M.: On the Gromov width of polygon spaces. Transform. Groups 23(1), 149-183 (2018)

[36] Pia, A., DelWeismantel, R.: On convergence in mixed integer programming. Math. Program. Ser. A 135(1-2), 397-412 (2012)

[37] Rudelson, M.: Distances between non-symmetric convex bodies and the $M M^{*}$ estimate. Positivity 4(2), 161-178 (2000)

[38] Schicho, J.: Simplification of surface parametrizations-a lattice polygon approach. J. Symb. Comput. 36(3-4), 535-554 (2003)

[39] Schlenk, F.: Embedding Problems in Symplectic Geometry, De Gruyter Expositions in Mathematics, vol. 40. Walter de Gruyter GmbH \& Co. KG, Berlin (2005)

[40] Sebö, A.: Hilbert bases, Caratheodorys theorem and combinatorial optimization. In: Proceedings of the IPCO Conference, Waterloo, Canada (1990)

[41] Schepers, J., Van Langenhoven, L.: Unimodality questions for integrally closed lattice polytopes. Ann. Comb. 17(3), 571-589 (2013)

Publisher's Note Springer Nature remains neutral with regard to jurisdictional claims in published maps and institutional affiliations. 